\title{
Highly porous and injectable hydrogels derived from cartilage acellularized matrix exhibit reduction and NIR light dual-responsive drug release properties for application in antitumor therapy
}

\author{
Muhammad Gulfam ${ }^{1,2}$, Sung-Han Jo ${ }^{3}$, Sung-Woo Jo ${ }^{1,2}$, Trung Thang Vu ${ }^{1,2}$, Sang-Hyug Park $\mathbb{D}^{3}$ and Kwon Taek Lim (1) ${ }^{1,2}$
}

\begin{abstract}
In this work, we developed novel stimuli-responsive injectable hydrogels composed of a highly biocompatible cartilage acellularized matrix (CAM) and a water-soluble cross-linker containing a diselenide bridge by using ultrafast norbornene ( $\mathrm{Nb}$ )-tetrazine $(\mathrm{Tz})$ click chemistry. The cross-linking reaction between the Nb groups of the CAM and Tz groups of the cross-linker evolved nitrogen gas and resulted in injectable hydrogels with highly porous structures. The synthesized hydrogels demonstrated high drug loading efficiencies (up to 93\%), good swelling ratios, and useful mechanical properties. The doxorubicin (DOX)-loaded hydrogels released minimal amounts of DOX in the simulated physiological medium; however, sustained release of DOX was detected under reducing conditions, revealing more than 90\% DOX release after $96 \mathrm{~h}$. Interestingly, the indocyanine green (ICG)-incorporated hydrogels produced reactive oxygen species upon exposure to NIR light and exhibited burst release ( $>50 \%$ DOX release) of DOX during the first $4 \mathrm{~h}$, followed by a sustained release phase. In vitro cytocompatibility tests showed that the synthesized CAM-Nb and hydrogels are essentially nontoxic to HFF-1 fibroblast cells and human colorectal adenocarcinoma cells (HT-29), indicating their excellent bioorthogonality and biocompatibility. Furthermore, DOX-loaded and DOX + ICG-loaded hydrogels inhibited the metabolic activities of HT-29 cells after GSH or NIR exposure and induced antitumor effects similar to those of free DOX. Therefore, these biocompatible and reduction-responsive injectable hydrogels, which exhibited on-demand drug release after NIR exposure, could be promising candidates for minimally invasive local delivery of cancer therapeutics.
\end{abstract}

\section{Introduction}

Cancerous tumors are fatal diseases owing to their antagonistic nature, leading to high mortality and morbidity rates worldwide ${ }^{1}$. Conventional treatment for this malignancy includes surgical intervention, chemotherapy, and radiotherapy ${ }^{2}$. Although chemotherapy has proven

\footnotetext{
Correspondence: Sang-Hyug Park (shpark1@pknu.ac.kr) or Kwon Taek Lim (ktlim@pknu.ac.kr)

${ }^{1}$ Department of Smart Green Technology Engineering, Pukyong National University, Busan 48513, Republic of Korea

${ }^{2}$ Department of Display Engineering, Pukyong National University, Busan 48513, Republic of Korea

Full list of author information is available at the end of the article

These authors contributed equally: Muhammad Gulfam, Sung-Han Jo.
}

broadly effective and remains the dominant modality in treating certain types of tumors, serious adverse effects, including nephrotoxicity, hepatotoxicity, compromised immune system, myelosuppression, destruction of normal cells, and drug resistance, have been reported ${ }^{3}$. Therefore, novel strategies to improve therapeutic efficacy and drug tolerance and to decrease the sequelae of cancer chemotherapy are urgently desired. To overcome these limitations, various controlled drug delivery systems, including nanoparticles, micelles, liposomes, and hydrogels, have been developed previously ${ }^{4}$. Specifically, hydrogels, with their 3D network, unique porous structure, high swelling ratio, good mechanical properties,

\section{(c) The Author(s) 2022}

\footnotetext{
(c) (i) Open Access This article is licensed under a Creative Commons Attribution 4.0 International License, which permits use, sharing, adaptation, distribution and reproduction cc) in any medium or format, as long as you give appropriate credit to the original author(s) and the source, provide a link to the Creative Commons license, and indicate if changes were made. The images or other third party material in this article are included in the article's Creative Commons license, unless indicated otherwise in a credit line to the material. If material is not included in the article's Creative Commons license and your intended use is not permitted by statutory regulation or exceeds the permitted use, you will need to obtain permission directly from the copyright holder. To view a copy of this license, visit http://creativecommons.org/licenses/by/4.0/.
} 
good biocompatibility, and capability to release the encapsulated therapeutic agent in a temporal pattern, have attracted great attention in tumor therapy ${ }^{5}$. Despite their advantages, conventional hydrogels with particular architectures typically have dosage limitations, and implanting them into a diseased organ/tissue requires an intrusive surgical process, which can be painful for patients and leads to lengthier recovery times and higher costs.

To address these barriers, injectable hydrogels, which could be administered in a minimally invasive process by a simple syringe injection to poorly accessible tissues, have attracted considerable attention in the last decade. These injectable hydrogels have been developed from synthetic or natural polymers by using various approaches, e.g., quick sol-gel phase transition ${ }^{6}$, in situ chemical polymerization ${ }^{7}$, and physical or chemical cross-linking strategies ${ }^{8}$. Specifically, naturally occurring extracellular matrix (ECM)-based biomaterials have become increasingly prevalent in the development of injectable hydrogels. The ECM is composed of structural and functional molecules produced by the intrinsic cells of each tissue and therefore exhibits low inflammatory responses, good biocompatibility, and inherent biodegradability. In addition, the ECM possesses various ligands, which promote cell adhesion and regulate cellular responses through binding with integrin receptors ${ }^{9}$. Despite several advantages, ECM and its hydrogels have relatively weak viscoelastic properties and rapid degradation rates ${ }^{10}$. The viscoelastic properties and degradation kinetics of ECM hydrogels have been previously tuned by chemical crosslinkers, such as formaldehyde, glutaraldehyde, genipin, and 1-ethyl-3-carbodiimide ${ }^{11}$. Although these agents have shown good cross-linking properties, several of them have been reported to exhibit high cytotoxicities ${ }^{12}$.

Recently, stimuli-responsive injectable hydrogels, which have the capability to sense variations in $\mathrm{pH}^{13}$, temperature $^{14}$, redox potential ${ }^{15}$, light ${ }^{16}$, electrical signals ${ }^{17}$, or enzymes $^{18}$ and respond by changing their shape or undergoing sol-gel transition, have been developed. Specifically, redox-responsive hydrogels, which exploit disease-specific redox variation as a trigger to prompt a diagnostic signal or to deliver a therapeutic molecule, have attracted considerable attention. In particular, these redox variations are significant aspects of tumor-specific pathological disorders, where redox potential is significantly heightened ( 10-100-fold) compared to that in healthy tissues ${ }^{19}$. These ranges of redox potential are of interest because injectable hydrogels in principle could be formulated to reciprocate differentially for tumor-specific drug delivery. Generally, the well-established chemistries for developing redox responses in injectable hydrogels are derived from disulfide bonds. In addition to disulfide bonds, it has been discovered that diselenide bonds undergo similar reversible cleavage upon exposure to a reduction environment ${ }^{20}$. Since $\mathrm{Se}-\mathrm{Se}$ and $\mathrm{C}-\mathrm{Se}$ bonds have lower energies (Se-Se $172 \mathrm{~kJ} / \mathrm{mol}, \mathrm{C}-\mathrm{Se} 244 \mathrm{~kJ} / \mathrm{mol}$ ) than S-S bonds $(268 \mathrm{~kJ} / \mathrm{mol})$, a fast responsive reductionsensitive hydrogel could be formulated by using diselenide bonds ${ }^{21}$.

In the past decade, combination therapy using NIR light-responsive organic and inorganic drug delivery systems has received substantial attention in cancer treatment. In general, organic NIR light-responsive drug delivery systems incorporate photosensitive compounds such as $o$-nitrobenzyl esters, coumarin and its derivatives, and indocyanine green dye (ICG) ${ }^{22}$. However, inorganic NIR light-responsive drug delivery systems mostly comprise antimony nanopolyhedrons ${ }^{23}$, selenium-coated tellurium nanoheterojunctions ${ }^{24}$, silicon, and black phosphorus nanostructures ${ }^{25,26}$, which efficiently convert optical energy into thermal energy and enhance the efficacy of photothermal therapy. In addition, diselenidebased drug delivery systems could also be used as NIR light-responsive drug delivery systems, as diselenide bonds are susceptible to reactive oxygen species (ROS) and can undergo reversible cleavage. ROS can be generated in situ by exposing near-infrared (NIR) light to a photosensitizer, such as an ICG (a clinically approved fluorophore). NIR light is harmless and can penetrate biological tissues deeply and noninvasively without damaging healthy tissues; therefore, the combination of ICG with diselenide bridges could increase the NIR sensitivity of these systems ${ }^{27}$. However, most of the reported diselenide-based molecules or cross-linkers suffer poor aqueous solubility and insufficient bioorthogonality, therefore necessitating the use of toxic organic solvents, catalysts, and additives, rendering these systems less suitable for bioorthogonal injectable hydrogels.

In this work, we developed reduction and NIR dualresponsive injectable hydrogels derived from cartilage acellularized matrix (CAM, a type of ECM) by using a novel diselenide-based and water-soluble cross-linker with excellent bioorthogonality and cytocompatibility. To achieve this goal, the CAM was functionalized with norbornene $(\mathrm{Nb})$ moieties, and then hydrogels were developed in one step by mixing CAM-Nb and various feed ratios of the cross-linker. The inverse electron-demand Diels-Alder (IEDDA) reaction between $\mathrm{Nb}$ and tetrazine (Tz) evolved nitrogen gas and resulted in injectable hydrogels with highly porous structures. To the best of our knowledge, this is the first report about the functionalization of CAM with $\mathrm{Nb}$ functionalities and the synthesis of Tz-conjugated reduction and NIR dualresponsive and water-soluble cross-linkers to formulate injectable and highly porous hydrogels. The data from the conducted experiments indicated that mechanical properties, porosity, swelling behavior, and drug release from 
the CAM-based injectable hydrogels could be controlled and that the synthesized hydrogels are nontoxic to the tested cell lines. Moreover, drug release from doxorubicin (DOX)-loaded hydrogels could be triggered upon exposure to GSH and NIR light, consequently inducing antitumor activity in colon cancer cells in vitro. The drug delivery system based on these dual-stimuli-responsive CAM hydrogels possesses a series of unique advantages. In the absence of endogenous and exogenous stimuli, this drug delivery system could achieve desired results, such as precise control over drug release and no leakage of loaded therapeutic molecules in the surrounding tissues, hence minimizing damage to healthy cells. In contrast, in the presence of internal (GSH) or external stimuli (NIR light), tumor-specific, robust, and on-demand drug delivery could be achieved to completely eradicate a tumor. Therefore, the developed reduction and NIR dualresponsive injectable hydrogels with highly porous structures and tunable properties could be promising candidates for dual-stimuli responsive drug delivery applications.

\section{Materials and methods}

\section{Materials, instruments, and measurements}

For materials, instruments, and measurements, see Supplementary information.

\section{Methods}

\section{Synthesis of CAM-Nb}

CAM was extracted using an established protocol with some modifications $^{28}$ (see SI, Section 3.1). Afterward, CAM was functionalized with $\mathrm{Nb}$ moieties by a two-step procedure. Briefly, 5-norbornene-2-carboxylic acid $(0.885 \mathrm{~mL}, 7.237 \mathrm{mmol}, 1 \mathrm{~mol}$. equi.) was dispersed in $20 \mathrm{~mL}$ of dry dichloromethane (DCM), and the contents were purged with nitrogen under constant stirring at $0{ }^{\circ} \mathrm{C}$. EDC. $\mathrm{HCl}(1.385,7.237 \mathrm{mmol}, 1 \mathrm{~mol}$ equi.) and NHS $(0.916 \mathrm{~g}, 7.960 \mathrm{mmol}, 1.1 \mathrm{~mol}$ equi.) were separately dissolved in dry DCM and introduced into the reaction by using a glass syringe. The reaction was stirred first for $1 \mathrm{~h}$ at $0^{\circ} \mathrm{C}$ and then at room temperature overnight. Afterward, the contents were diluted with $100 \mathrm{~mL}$ of DCM, washed with $3 \times 100 \mathrm{~mL}$ brine, dried with $\mathrm{MgSO}_{4}$ (anhydrous), filtered, and dried using a rotavap to obtain an $\mathrm{Nb}$ NHS active ester ( $89 \%$ yield). To functionalize the CAM with $\mathrm{Nb}$ functionalities, CAM powder $(2.76 \mathrm{~g}$, $\sim 0.0184 \mathrm{mmol}$ ) was dispersed in $270 \mathrm{~mL}$ of bicarbonate buffer ( $\mathrm{pH} 9$ ) and stirred until a homogenous suspension was obtained. Nb-NHS active ester solution in $30 \mathrm{~mL}$ of DMSO $(0.820 \mathrm{~g}, 3.40 \mathrm{mmol}, 50 \mathrm{~mol} \%$ of free amine contents of CAM) was introduced to this solution. The suspension was stirred for $48 \mathrm{~h}$ at room temperature and then quenched with hydroxylamine hydrochloride $(236.3 \mathrm{mg}, 3.4 \mathrm{mmol})$. The suspension was precipitated with 5 volumes of ethanol, filtered under vacuum and dried in a vacuum oven at $30^{\circ} \mathrm{C}$. Finally, the solid CAM was again suspended in $50 \mathrm{~mL}$ of DI water and dialyzed for $48 \mathrm{~h}$ by using a dialysis membrane (MWCO $\sim 14 \mathrm{kDa}$ ). Finally, the suspension was lyophilized to obtain CAM$\mathrm{Nb}$. The degree of substitution of CAM-Nb was determined by a ninhydrin assay (SI, Section 2.7). The calculated degree of substitution was $32 \%$ of the original amine content.

\section{Synthesis of di-selenide-di-polyethylene glycol (DSe-DPEG)}

In a typical procedure, black selenium powder $(0.375 \mathrm{~g}$, $4.75 \mathrm{mmol}$ ) was dispersed in $20 \mathrm{~mL}$ of DI water in a 3-neck RBF equipped with a glass condenser and an ice bath. The reaction mixture was deoxygenized by nitrogen gas for $10 \mathrm{~min}$ at $0^{\circ} \mathrm{C}$. Sodium borohydride $(0.359 \mathrm{~g}$, $9.51 \mathrm{mmol}$, pre-dissolved in $10 \mathrm{~mL}$ of cold DI water) was carefully added dropwise into the reaction flask, and the contents were stirred for $30 \mathrm{~min}$ at $0^{\circ} \mathrm{C}$ or until the contents had completely subsided and the selenium was dissolved. Afterward, another quantity of selenium pow$\operatorname{der}(0.375 \mathrm{~g}, 4.75 \mathrm{mmol})$ was introduced into the reaction flask, and the contents were stirred for $1.5 \mathrm{~h}$ at $100^{\circ} \mathrm{C}$ while continuously passing nitrogen through the reactor (the system must not be closed because it will generate a toxic $\mathrm{H}_{2} \mathrm{Se}$ gas that should be expelled from the system). At the end of the reaction, the color turned reddishbrown (a typical sign of $\mathrm{Na}_{2} \mathrm{Se}_{2}$ formation). Subsequently, PEG-OTs $(5.805 \mathrm{~g}, 10.47 \mathrm{mmol}$, see SI, Section 3.2 for synthesis protocol) were dissolved in $20 \mathrm{~mL}$ of DI water, basified ( $\mathrm{pH}$ 8) with $\mathrm{Na}_{2} \mathrm{CO}_{3}$ and introduced into the $\mathrm{Na}_{2} \mathrm{Se}_{2}$ solution. The contents were stirred under an inert atmosphere overnight, opened to air for $1 \mathrm{~h}$, filtered, and extracted with $2 \times 100 \mathrm{~mL}$ DCM. The organic phases were collected and washed with $3 \times 100 \mathrm{~mL}$ of DI water and then with $2 \times 100 \mathrm{~mL}$ of brine and dried with $\mathrm{MgSO}_{4}$, and the solvent was removed under vacuum. Finally, the paleyellow solid was first recrystallized in cold ether and then further purified by flash column chromatography $(30 \%$ methanol in ethyl acetate, $R_{\mathrm{f}}=0.56$, yield $=57 \%$, yellow solid). ${ }^{1} \mathrm{H}$ NMR (400 MHz, DMSO- $\left.d_{6}, \delta\right): 4.60(\mathrm{t}, 2 \mathrm{H})$, $3.66(\mathrm{t}, 4 \mathrm{H}), 3.50(\mathrm{~m}, 173 \mathrm{H}), 3.10(\mathrm{t}, 4 \mathrm{H})$.

\section{Synthesis of di-selenide-di-polyethylene glycol-di-tetrazine (DSe-DPEG-DTz)}

Tetrazine(benzylamino)-5-oxopentanoic acid (Tz$\mathrm{COOH}, 0.20 \mathrm{~g}, 0.663 \mathrm{mmol}, 2.5 \mathrm{~mol}$ equi., see SI methods for synthesis protocol) was dissolved in a mixture of DCM and DMSO (9:1 ratio) at $0{ }^{\circ} \mathrm{C}$ and purged with nitrogen for $30 \mathrm{~min}$. DMAP $(0.081 \mathrm{~g}, 0.663 \mathrm{mmol}, 2.5 \mathrm{~mol}$ equi.) was dissolved in a minimum quantity of DCM and introduced into the system by using a glass syringe. Next, EDC. $\mathrm{HCl}(0.1272 \mathrm{~g}, 0.663 \mathrm{mmol}, 2.5 \mathrm{~mol}$ equi., dissolved in a minimum quantity of DCM) was added, and the 
contents were stirred for $1 \mathrm{~h}$ to form an active ester. Subsequently, DSe-DPEG $(0.572 \mathrm{~g}, 0.265 \mathrm{mmol}, 1 \mathrm{~mol}$ equi.) was dissolved in $5 \mathrm{~mL}$ of DCM and injected into the reactor with the help of a glass syringe. The contents were stirred under an inert atmosphere for $48 \mathrm{~h}$ at room temperature. Subsequently, the reaction mixture was diluted with DCM $(100 \mathrm{~mL})$, washed with $3 \times 100 \mathrm{ml}$ of DI water, and then with $100 \mathrm{~mL}$ of brine and dried with $\mathrm{MgSO}_{4}$, and the solvent was removed by a rotavap. Finally, the compound was purified by column chromatography $(5 \%$ methanol in DCM, isolated yield $=59 \%) .{ }^{1} \mathrm{H}$ NMR $\left(400 \mathrm{MHz}, \mathrm{DMSO}-d_{6}, \delta\right): 10.58(\mathrm{~s}, 2 \mathrm{H}), 8.48(\mathrm{~s}, 4 \mathrm{H}), 7.56$ $(\mathrm{s}, 4 \mathrm{H}), 4.40(\mathrm{~d}, 4 \mathrm{H}) 4.13(\mathrm{t}, 4 \mathrm{H}), 3.68(\mathrm{t}, 4 \mathrm{H}), 3.52(\mathrm{~m}$, $173 \mathrm{H}), 3.10(\mathrm{t}, 4 \mathrm{H}), 2.33(\mathrm{t}, 4 \mathrm{H}), 2.24(\mathrm{t}, 4 \mathrm{H}), 1.81(\mathrm{t}, 4 \mathrm{H})$.

\section{Preparation of injectable hydrogels}

CAM-based injectable and porous hydrogels were prepared by reacting $\mathrm{Nb}$ groups of the $\mathrm{CAM}-\mathrm{Nb}$ and $\mathrm{Tz}$ groups of the DSe-DPEG-DTz cross-linker. Briefly, to a well-mixed solution (in glass vials) containing $2 \%$ or $4 \% \mathrm{CAM}-\mathrm{Nb}$ in PBS, DSe-DPEG-DTz cross-linker (dissolved in PBS) was added according to various mol. ratios $(10: 10$ or $10: 5, \mathrm{Nb}$ to $\mathrm{Tz}$ ratios, respectively), as shown in Table 1 . The solutions were gently mixed by using a vortex for $10 \mathrm{~s}$ and were immediately transferred to an injection syringe equipped with a 25-gauge needle to test the injectability. The gelation times of hydrogels were recorded using an inverted vial/tube test with the help of a digital stopwatch. Similarly, DOXloaded or DOX + ICG-loaded hydrogels were prepared by mixing 1 or $2 \mathrm{mg} / \mathrm{mL}$ DOX (for $2 \%$ or $4 \%$ CAM $\mathrm{Nb}$, respectively) with or without $1 \mathrm{mg} / \mathrm{mL}$ ICG in CAM-Nb solution prior to the addition of the cross-linker, as described above. To analyze the porous structures of hydrogels, rhodamine-G6 (as a standard fluorescent dye) was also incorporated into the CAM-Nb solutions prior to the addition of the cross-linker. The porous structures of injectable hydrogels were assessed by confocal laser microscopy and further confirmed by field-emission electron microscopy (FESEM). The viscoelastic properties of hydrogels were analyzed by a rheometer (see SI for further details).

\section{Measurement of loading efficiency}

The DOX loading efficiency was measured by washing the DOX-loaded hydrogels with $3 \mathrm{~mL}$ of PBS, and the quantity of DOX in the supernatant was calculated by using UV-Vis. spectrophotometry $\left(\lambda_{\max }=485 \mathrm{~nm}\right)$ and obtaining a standard curve of DOX in PBS. The drug loading efficiency (\%) was measured according to the following equation:

$$
\begin{aligned}
& \text { Drug loading efficiency }(\%)= \\
& \frac{\text { (quantity of DOX in feed }- \text { quantity of DOX in supernatant) }}{\text { (quantity of DOX in feed) }} \times 100
\end{aligned}
$$

\section{Drug release assessment}

The in vitro drug release assessments from DOX-loaded hydrogels were performed in a simulated physiological medium (PBS, pH 7.4) and reducing (10 mmol GSH in PBS) medium. Additionally, drug release experiments were carried out after exposure to NIR light for $15 \mathrm{~min}$ at 2-watt power. For NIR-responsive release studies, DOX + ICGloaded hydrogels were used in PBS (pH 7.4). For all drug release experiments, lyophilized disk-shaped hydrogels were immersed in $5 \mathrm{~mL}$ of the corresponding medium in a dialysis bag ( $3.5 \mathrm{~K} \mathrm{MWCO})$ and subsequently dialyzed against $30 \mathrm{~mL}$ of release medium at $37^{\circ} \mathrm{C}$ and $100 \mathrm{rpm}$. Aliquots $(2 \mathrm{~mL})$ were taken at predetermined time intervals and replaced with $2 \mathrm{~mL}$ of fresh medium. The cumulative amount of released DOX was calculated using UV-Vis. spectrophotometry $\left(\lambda_{\max }=485 \mathrm{~nm}\right)$ via a standard curve of DOX in PBS or in $10 \mathrm{mmol} \mathrm{GSH}$.

\section{In vitro cytocompatibility of CAM, CAM-Nb, and hydrogels}

The cytocompatibilities of CAM and CAM-Nb precursors were studied in a human fibroblast cell line (HFF1 cells) by using WST assays (EZ-cytox, South Korea). For this purpose, HFF-1 cells were cultured on a 48-well plate at a density of 10,000 cells/well in $500 \mu \mathrm{L}$ of Dulbecco's modified Eagle's medium (DMEM, GE Healthcare, Boston, MA, USA) containing 10\% fetal bovine serum (FBS, GIBCO, USA) and $1 \%$ antibiotic-antimycotic (Gibco, USA) at $37^{\circ} \mathrm{C}$ with $5 \% \mathrm{CO}_{2}$ for $24 \mathrm{~h}$. Afterward, the cell culture medium was aspirated and replaced with a new medium containing various amounts $(100,500,1000$, 1500 , and $2000 \mu \mathrm{g} / \mathrm{mL}$ ) of CAM or CAM-Nb. Cells were incubated for $48 \mathrm{~h}$ and then washed two times with $100 \mu \mathrm{L}$ of PBS. Subsequently, $10 \mu \mathrm{L}$ of WST assay solution

Table 1 Formulations of CAM hydrogels, gelation times, drug loading efficiencies, and drug loading contents.

\begin{tabular}{llllll}
\hline Hydrogel code & CAM \% & Mol. feed ratio (Nb: Tz) & Gelation time (s) & Drug loading efficiency (\%) & Drug loading contents (\%) \\
\hline CAMHG-1 & 2 & $10: 5$ & $478 \pm 30$ & $73 \pm 6$ & $3.65 \pm 0.07$ \\
CAMHG-2 & 2 & $10: 10$ & $121 \pm 15$ & $81 \pm 5$ & $4.05 \pm 0.04$ \\
CAMHG-3 & 4 & $10: 5$ & $72 \pm 20$ & $89 \pm 2$ & $4.51 \pm 0.06$ \\
CAMHG-4 & 4 & $10: 10$ & $32 \pm 20$ & $93 \pm 3$ & $4.64 \pm 0.05$ \\
\hline
\end{tabular}


was added to each well. The optical densities at $450 \mathrm{~nm}$ were recorded using a microplate reader. The biocompatibility of the CAM-Nb precursor was further confirmed by a calcein AM/ethidium bromide kit. For this purpose, HFF-1 cells (cultured on confocal microscopy dishes) were treated with various amounts of CAM-Nb for $48 \mathrm{~h}$. Afterward, the cells were immunostained with calcein-AM to detect live cells and counterstained with ethidium bromide to detect dead cells. Similarly, the cytocompatibilities of empty hydrogels were assessed in human colorectal adenocarcinoma cells (HT-29) by using WST assays, as described above.

\section{In vitro antitumor activity of DOX-loaded/DOX + ICG-loaded hydrogels}

The antitumor activity or cancer cell inhibition effects of DOX-loaded hydrogels were assessed in HT-29 cells as a model cancer cell line by using WST assays. To assess GSH-responsive DOX release and consequent antitumor activity, HT-29 cells were cultured on a 48-well plate at a density of 10,000 cells/well in $500 \mu \mathrm{L}$ of DMEM containing $10 \% \mathrm{FBS}$ and antibiotics at $37^{\circ} \mathrm{C}$ with $5 \% \mathrm{CO}_{2}$ for $24 \mathrm{~h}$. Afterward, the cell culture medium was removed, and the cells were incubated with a fresh medium containing $10 \mathrm{mmol} \mathrm{GSH}$. After $1 \mathrm{~h}$, the GSH-containing medium was aspirated, and the cells were treated with DOX-loaded hydrogels and free DOX for $48 \mathrm{~h}$. The antitumor activity of DOX-loaded hydrogels and free DOX was calculated by using WST assays as described above. For NIR lighttriggered DOX release and the resulting antitumor activity assessment, HT-29 cells were seeded on Transwell culture plates (bottom chamber), and free DOX- and DOX + ICG-loaded hydrogels were placed on the top chamber of the Transwell culture plates. The samples were irradiated with a $2 \mathrm{~W}$ NIR laser for $90 \mathrm{~s}$. Afterward, the cells were incubated for $48 \mathrm{~h}$. The antitumor activity of the samples was calculated by using WST assays as described above.

\section{Results and discussion}

\section{Preparation and characterization of CAM and CAM-Nb}

To preserve the intrinsic properties of the CAM, we designed an optimized protocol (Fig. 1A) that effectively removed the tissue components/cells (cartilage cells and blood cells), nuclei, and any other genetic materials while preserving or minimally altering the native collagen and glycosaminoglycan (GAG) contents of the CAM, as shown in Fig. 1B. Specifically, the processed CAM powder retained $400 \mu \mathrm{g}$ of collagen (Fig. 1B-a) and $175 \mu \mathrm{g}$ GAGs (Fig. 1B-b) per milligram of CAM, indicating that the extracted CAM powder retained almost all the collagen contents and $75 \%$ of the glycosaminoglycan contents compared to the native cartilage matrix. Moreover, DNA was not detected, indicating immaculate elimination of all genetic materials (Fig. 1B-c). SDS-PAGE analysis demonstrated collagen proteins with a dominant molecular weight of $150 \mathrm{kDa}$ (Fig. 1B-d), which was in good agreement with previously reported protocols ${ }^{29}$. Overall, the CAM powder was successfully prepared as a biomaterial for the development of CAM-based hydrogels.

To develop a bioorthogonally clickable CAM precursor for the IEDDA click reaction, CAM was functionalized with $\mathrm{Nb}$ moieties via the reaction of primary amines of CAM with the carboxylic acid functionality of 5norbornene-2-carboxylic acid by means of a carbodiimide coupling reaction. Prior to this, the free amine contents of CAM were determined by a ninhydrin assay using glycine as a standard amino acid, which was found to be $4 \mathrm{wt} \%$ (Fig. S1). Notably, during the functionalization procedure, direct addition of EDC or its unreacted residues resulted in the creation of zero-length cross-linking bridges between the primary amines and the carboxylic acids, rendering the CAM insoluble in aqueous medium (data not shown), as reported previously ${ }^{30}$. To prevent this self-cross-linking, we adopted a two-step functionalization procedure (Fig. 1C-a). First, 5-norbornene-2-carboxylic acid was converted into its NHS active ester, and after complete removal of the EDC and NHS residues, it was allowed to react with the CAM suspension in a basic medium to afford CAM-Nb. The synthesis of CAM-Nb was confirmed by FTIR (Fig. 1C-b), and the degree of substitution was determined by a ninhydrin assay (Fig. S1) and found to be $32 \%$ of the original amine contents. From the perspective of preserving CAM bioactivity after chemical functionalization, this degree of substitution might be suitable, as the majority of the original bioactive primary amine functionalities remain available for cell recognition. Overall, CAM-Nb was successfully prepared as a novel precursor of hydrogels and other biomaterials.

\section{Synthesis and characterization of the diselenide- containing precursor (DSe-DPEG)}

Selenium, as an antioxidant and therapeutic agent, has attracted considerable attention for biotherapy and drug delivery $^{31}$. In particular, diselenide is a useful derivative of selenium that can undergo reversible cleavage in the presence of either ROS or reducing stimuli ${ }^{21}$. To prepare a water-soluble redox-responsive diselenide-containing cross-linker, we first developed bifunctional PEG ( $\alpha$-tosyl$\omega$-hydroxy polyethylene glycol, PEG-OTs), a highly potent intermediate, by reacting an equimolar amount of PEG with $p$-toluenesulfonyl chloride, as shown in Scheme 1A. The unreacted PEG was removed by a liquid-liquid extraction method, whereas bitosylated PEG was removed by using flash column chromatography. The chemical shift at $2.46 \mathrm{ppm}$ in the ${ }^{1} \mathrm{H}$ NMR spectrum of PEG-OTs in deuterated chloroform (Fig. S2) corresponded to the methyl protons of the tosyl group, whereas the chemical shift at $4.17 \mathrm{ppm}$ corresponded to the methylene protons of 


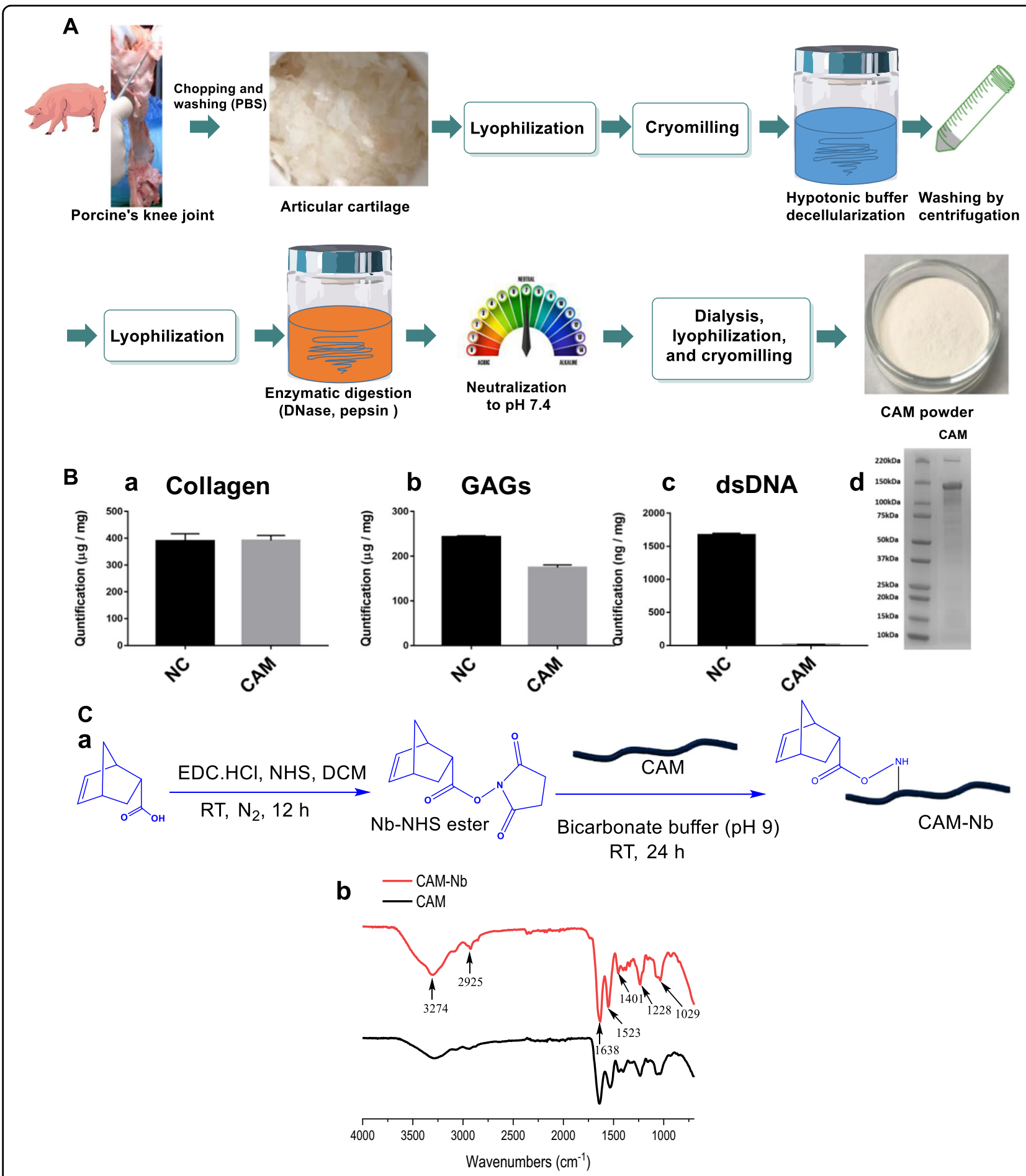

Fig. 1 Synthesis and characterization of cartilage acellularized matrix (CAM) and CAM-Nb. A Schematic representation of the isolation of CAM from porcine knee joints, B characterization of CAM for remaining (a), collagen, (b) glycosaminoglycans (GAGs), (c) double-stranded DNA (dsDNA), and (d) molecular weight assessment by using SDS-page test, and $\mathbf{C}$ synthesis and characterization of CAM-Nb, (a) scheme showing postextraction functionalization of CAM with 5-norbornene-2-carboxylic acid, and (b) ATR-FTR characterization of CAM and CAM-Nb. 


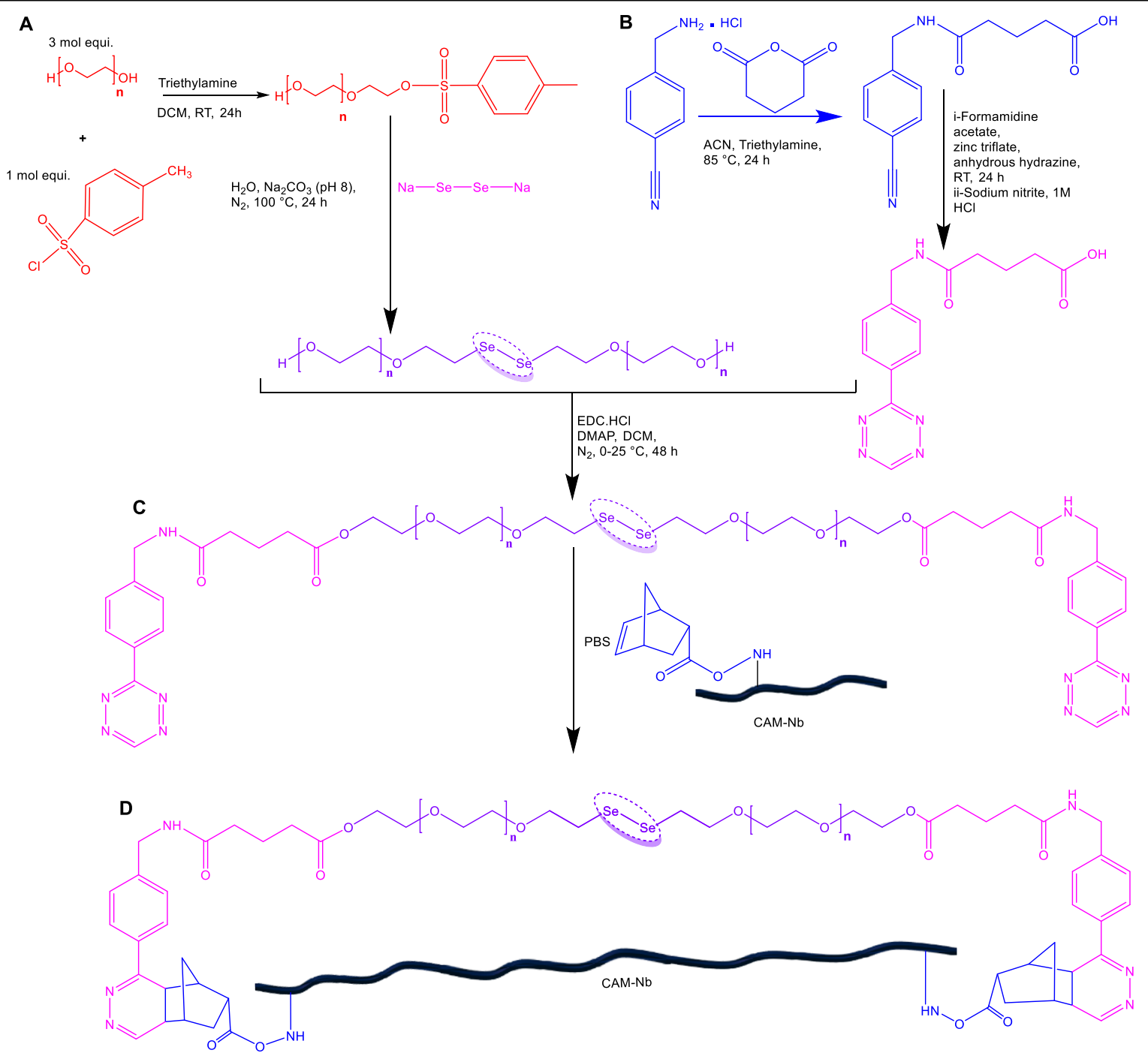

Scheme 1 Schematic illustration of the syntheses of A DSe-DPEG, B tetrazine-carboxylic acid, $\mathbf{C}$ redox, and NIR dual responsive water-soluble DSeDPEG-DTz cross-linker, and $\mathbf{D}$ reaction mechanism and chemical structure of the porous and injectable CAM-based hydrogels developed by the IEDDA click reaction.

PEG next to the tosyl group. The chemical shifts at 7.37 and $7.82 \mathrm{ppm}$ corresponded to the aromatic protons of the tosyl group. These signals confirmed the synthesis of PEGOTs. The monotosylated nature of the PEG-OTs was confirmed by performing ${ }^{1} \mathrm{H}$ NMR spectroscopy in deuterated DMSO (Fig. 2A). The chemical shift corresponding to hydroxyl protons was observed at $4.57 \mathrm{ppm}$, which was well separated from the PEG backbone peaks and did not broaden or shift with variation in the concentration, showing quantitative conversion of PEG to PEG-OTs. In a subsequent step, this asymmetric PEG derivative reacted with sodium diselenide ${ }^{32}$ to afford DSe-DPEG, as illustrated in Scheme 1A. The appearance of new chemical shifts at $3.10 \mathrm{ppm}\left(4 \mathrm{H}, \mathrm{CH}_{2}-\mathrm{Se}-\mathrm{Se}-\mathrm{CH}_{2}\right)$ and at $3.68 \mathrm{ppm}$ $\left(4 \mathrm{H},-\mathrm{O}-\mathrm{CH}_{2}-\mathrm{CH}_{2}-\mathrm{Se}-\mathrm{Se}-\mathrm{CH}_{2}-\mathrm{CH}_{2}-\mathrm{O}-\right)$ in the ${ }^{1} \mathrm{H}$
NMR spectrum (Fig. 2B) indicated the successful incorporation of diselenide linkages within the two PEG spacers. Additionally, DSe-DPEG was characterized by a GPC/SEC (SI, Fig. S3) and showed a decrease in the retention time of DSe-DPEG compared to those of PEG and PEG-OTs, indicating a higher molecular weight of DSe-DPEG than of the starting materials, confirming the synthesis of DSeDPEG without any apparent degradation.

\section{Synthesis and characterization of aqueous soluble diselenide-containing bioorthogonally clickable cross- linkers (DSe-DPEG-DTz)}

The DSe-DPEG-DTz cross-linker for injectable hydrogels was synthesized by using an asymmetric tetrazine derivative in the IEDDA reaction. For this purpose, the 


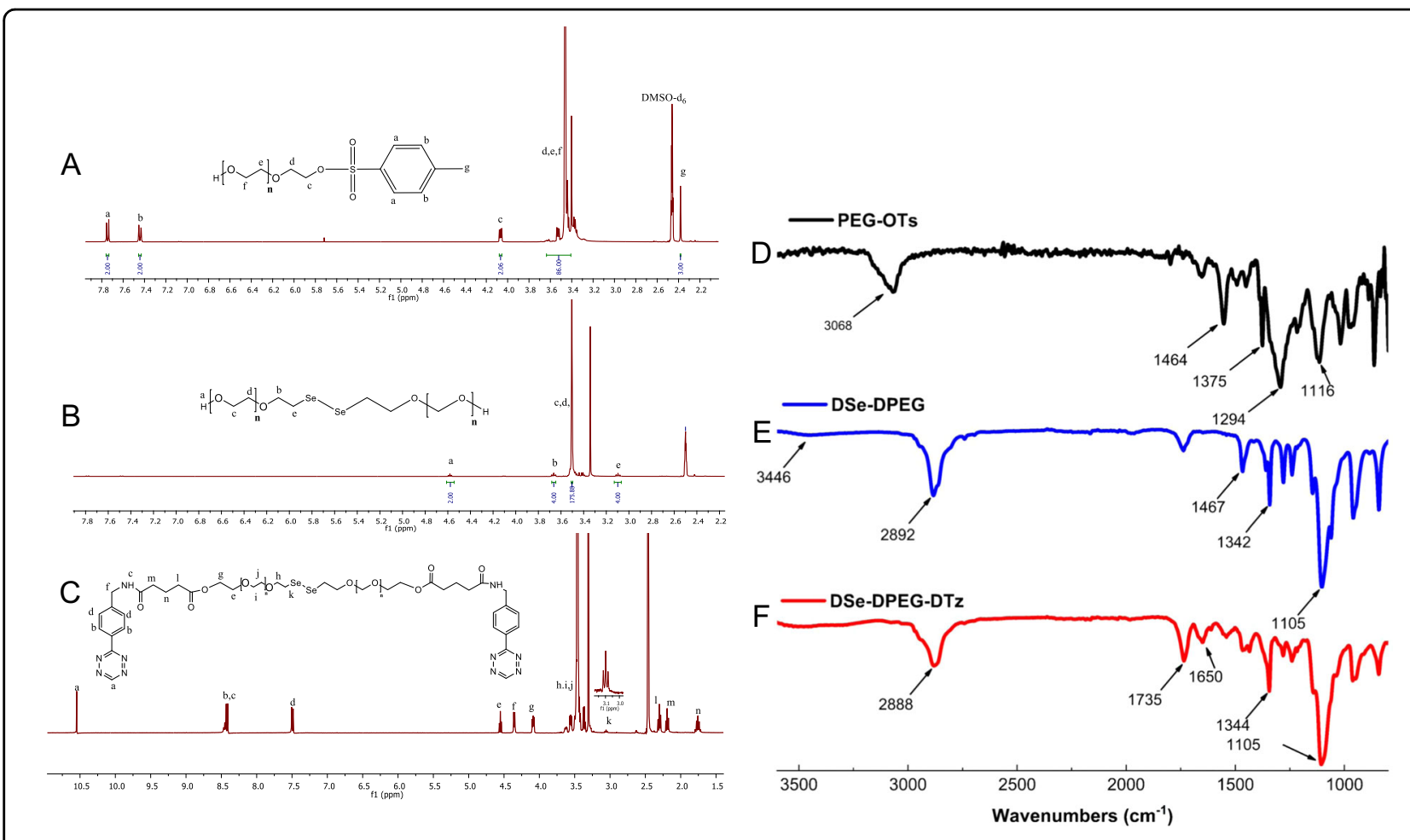

Fig. 2 Characterization of synthesized precursors and the cross-linker. A-C ${ }^{1} H$ NMR spectra of monotosylated PEG (PEG-OTS), DSe-DPEG, and DSE-DPEG-DTz cross-linkers. D-F ATR-FTIR spectra of synthesized precursors and the cross-linker.

asymmetric tetrazine derivative (5-(4-(cyano) benzylamino)-5-oxopentanoic acid, Figs. S4, 5 and Scheme 1B) was conjugated to DSe-DPEG by creating ester linkages between the carboxylic acid group of the asymmetric tetrazine derivative and two hydroxyl groups of DSeDPEG via carbodiimide chemistry, yielding a highly water-soluble and dual-responsive cross-linker (Scheme 1C). The structure of this cross-linker was analyzed by ${ }^{1} \mathrm{H}$ NMR (Fig. 2C) and showed a new chemical shift at $4.13 \mathrm{ppm}$, which represents the protons adjacent to ester linkages, and chemical shifts of the PEG backbone appeared at $3.51 \mathrm{ppm}$. However, all the other chemical shifts corresponding to $\mathrm{Tz}$ and DSe-DPEG remained intact. The structures of the starting materials and the cross-linker were further confirmed by ATR-FTIR (Fig. 2D-F), which showed a characteristic stretching peak of carbonyl carbons at $1735 \mathrm{~cm}^{-1}$, demonstrating the successful formation of the DSe-DPEG-DTz cross-linker for bioorthogonal click chemistry. Bioorthogonal click chemistry has received much attention over the last decade for developing injectable hydrogels. Preferably, these bioorthogonal reactions should be highly efficient and selective and should progress in aqueous medium at physiological $\mathrm{pH}$ and temperature ${ }^{33}$. However, not all bioorthogonal click reactions satisfy all the requirements of being entirely bioorthogonal. For instance, the requirement of copper for azide-alkyne Huisgen cycloaddition raises cytotoxicity issues, whereas strainpromoted azide-alkyne cycloaddition (copper-free click reaction) lacks complete selectivity, as the cyclooctyne moiety may react with cellular and plasma nucleophiles ${ }^{34}$. Therefore, the synthesized IEDDA clickable crosslinker could satisfy most of the bioorthogonality criteria.

\section{Preparation and characterization of highly porous dual- responsive injectable hydrogels Preparation of hydrogels and gelation behavior}

We prepared injectable CAM hydrogels via an IEDDA click reaction between the $\mathrm{Tz}$ groups of the diselenidebased aqueous soluble cross-linker and the norbornene group of CAM-Nb (Fig. S6 and Scheme 1D). Specifically, injectable hydrogels were prepared by the one-step mixing of various concentrations ( $2 \%$ or $4 \%$ ) of CAM-Nb with different feed ratios of the DSe-DPEG-DTz cross-linker (10:10 or 10:5 mol. ratio of $\mathrm{Nb}$ to $\mathrm{Tz}$, see Table 1$)$ at room temperature. The gelation times of the hydrogels were recorded by using an inverted vial/tube test. It is worth noting that gelation times were influenced by the concentration of the precursor (CAM-Nb) as well as by the molar feed ratio of the cross-linker. Specifically, CAMHG1 (10:5, Nb:Tz ratio) showed a gelation time of $\sim 8 \mathrm{~min}$, which decreased to $2 \mathrm{~min}$ when the feed ratio of the crosslinker was increased to 10:10 (CAMHG-2). A similar gelation behavior was observed with hydrogels made from 
4\% CAM-Nb. In particular, CAMHG-3 (10:5 mol. ratio of $\mathrm{Nb}: \mathrm{Tz}$ ) showed a gelation time of $\sim 1.2 \mathrm{~min}$, whereas the gelation behavior of CAMHG-4 hydrogels was relatively rapid ( $\sim .5 \mathrm{~min})$. From these findings, it could be noted that the gelation times of the injectable hydrogels could be tuned by adjusting the concentration of the CAM-Nb precursor and the feed ratio of the DSe-DPEG-DTz crosslinker. These gelation time ranges demonstrated that the synthesized hydrogels are injectable, which could not only provide adequate time for formulation, mixing, and injection of the pregel solution into the body but could also help to fill the irregular shapes of tissues or organs after injection, followed by solid gel formation ${ }^{35}$.

\section{Morphology of hydrogels}

Injectable CAM hydrogels with porous structures may offer several benefits in drug delivery, tissue engineering, and regenerative medicine. The developed CAM-based injectable hydrogels showed highly porous morphologies (Fig.
3A), which could be attributed to the nitrogen gas generated from the cross-linking reaction, leading to the in situ formation of microbubbles within the hydrogels ${ }^{36}$. Specifically, the IEDDA reaction between the electron-poor $\mathrm{Tz}$ (diene) and the electron-rich $\mathrm{Nb}$ (dienophiles) takes place quite vigorously under physiological environments and creates sturdy covalent links with the release of gaseous nitrogen as a solo inert byproduct, which creates pores inside the hydrogels. The in situ porous architectures generated by the bubble formation process was visualized by confocal laser scanning microscopy (without lyophilization) using rhodamine-G6 as a standard fluorescent dye. The confocal micrographs revealed that all the formulated hydrogels possess apparently porous structures, as shown by the red bubbles in the hydrogels (Fig. 3B). Interestingly, the porosity of the hydrogels (without lyophilization) improved by increasing the concentrations of the cross-linker.

The morphologies of CAM hydrogels were also analyzed by FE-SEM (Fig. 3C, D) to examine the porosity after lyophilization, where internal water droplets and nitrogen

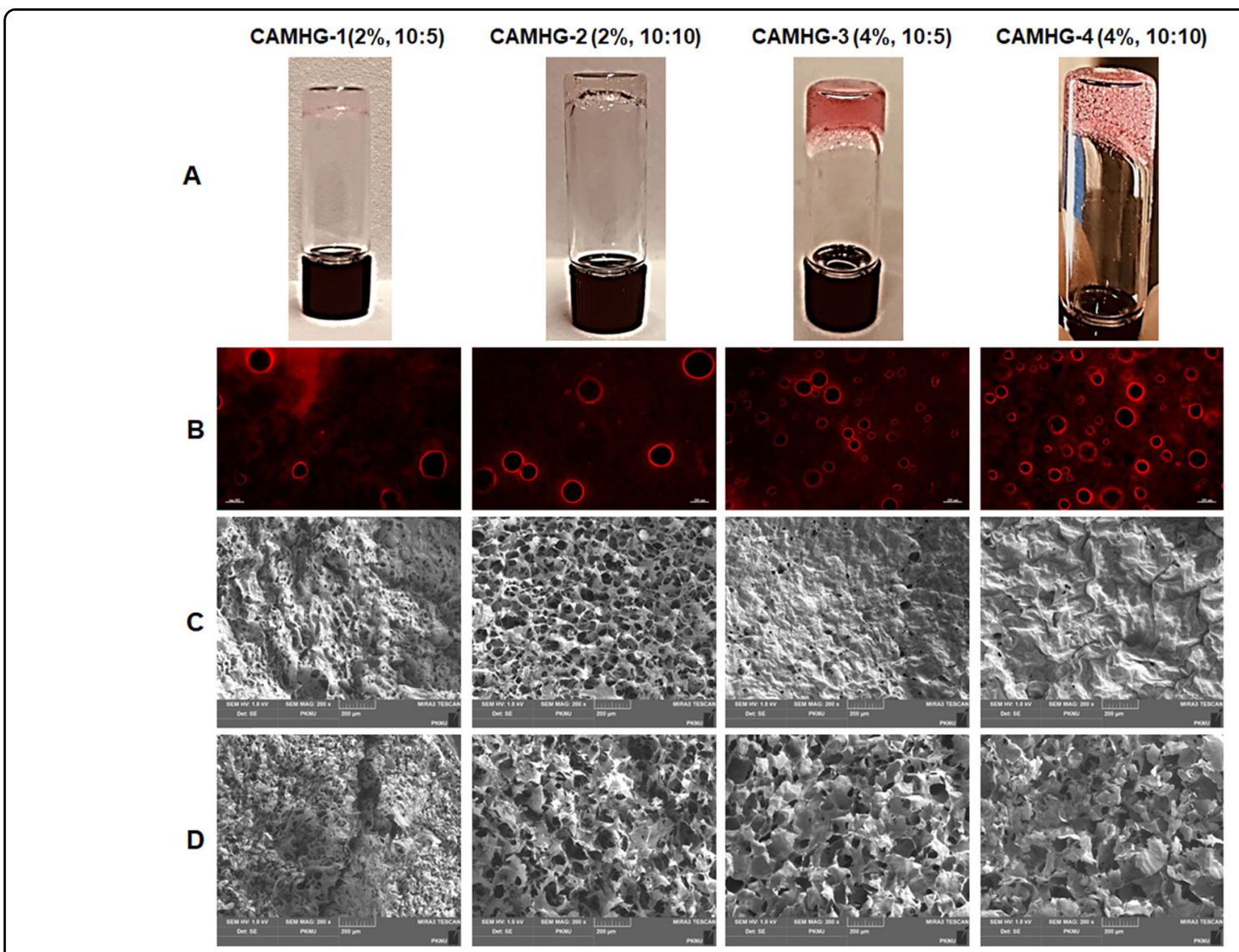

Fig. 3 Morphological characterization of CAM hydrogels. A Photographs of synthesized hydrogels, B confocal laser scanning micrographs of rhodamine-6G loaded hydrogels without lyophilization, C FE-SEM micrographs of hydrogels showing surface morphology after lyophilization, and $\mathbf{D}$ FE-SEM micrographs of hydrogels obtained from the cross-sectional area. Error bars showing $200 \mu \mathrm{m}$. Ratios represent the molar feed ratio of $\mathrm{Nb}$ to Tz. 
bubbles were leached out. It was revealed that the surface porosity of hydrogels decreased when the concentration of CAM-Nb was increased (from $2 \%$ to $4 \%$ ), which could be attributed to the highly dense polymer chains resulting in the reduction in mesh sizes. However, the internal structures of all the hydrogels were highly porous, as shown by the FESEM images (Fig. 3D) obtained from the cross-sections of hydrogels. The porosity in injectable hydrogels can be vital for drug release, cell infiltration, nutrients, and effective oxygen supply, and waste diffusion in a 3D microenvironment, facilitating the creation of functional tissues ${ }^{37}$. In general, porosity in injectable hydrogels has been engineered by using cytotoxic ingredients in the preparatory phases and/or by using destructive methods (e.g., emulsion template, lyophilization, porogen pore-forming, etc. $)^{38}$, which are unfavorable for injectable hydrogels, and excludes any likelihood of injecting the hydrogel into the body. Therefore, porous and injectable hydrogels developed by the in situ nitrogen generation process without using any additional additives or catalysts could be beneficial.

\section{Rheological properties of hydrogels}

In general, native ECM and its hydrogels possess poor mechanical properties. Therefore, assessment of the mechanical strength and subsequent reinforcement are among the desirable accomplishments in the engineering of CAM-based hydrogels. We evaluated the mechanical strength of CAM hydrogels by recording their storage modulus $\left(G^{\prime}\right)$ and loss modulus $\left(G^{\prime \prime}\right)$ and plotted their values as a function of time and angular frequency (Fig. 4). The time sweep graphs (Fig. 4A-D) demonstrated that during the cross-linking of CAM chains, the $G^{\prime}$ values of all four types of hydrogels gradually increased and reached a plateau after $\sim 10 \mathrm{~min}$, showing that gel formation was complete. In hydrogels prepared from $2 \%$ CAM-Nb (CAMHG-1, CAMHG-2), G" was larger than $G^{\prime}$ at the beginning, which was reasonable since the mixtures were still in a sol-gel state. As the formulations started to change into a gel-like structure, both moduli were amplified. Nonetheless, the rate of increase of $G^{\prime}$ was greater than that of $G^{\prime \prime}$ once the elastic behavior began to
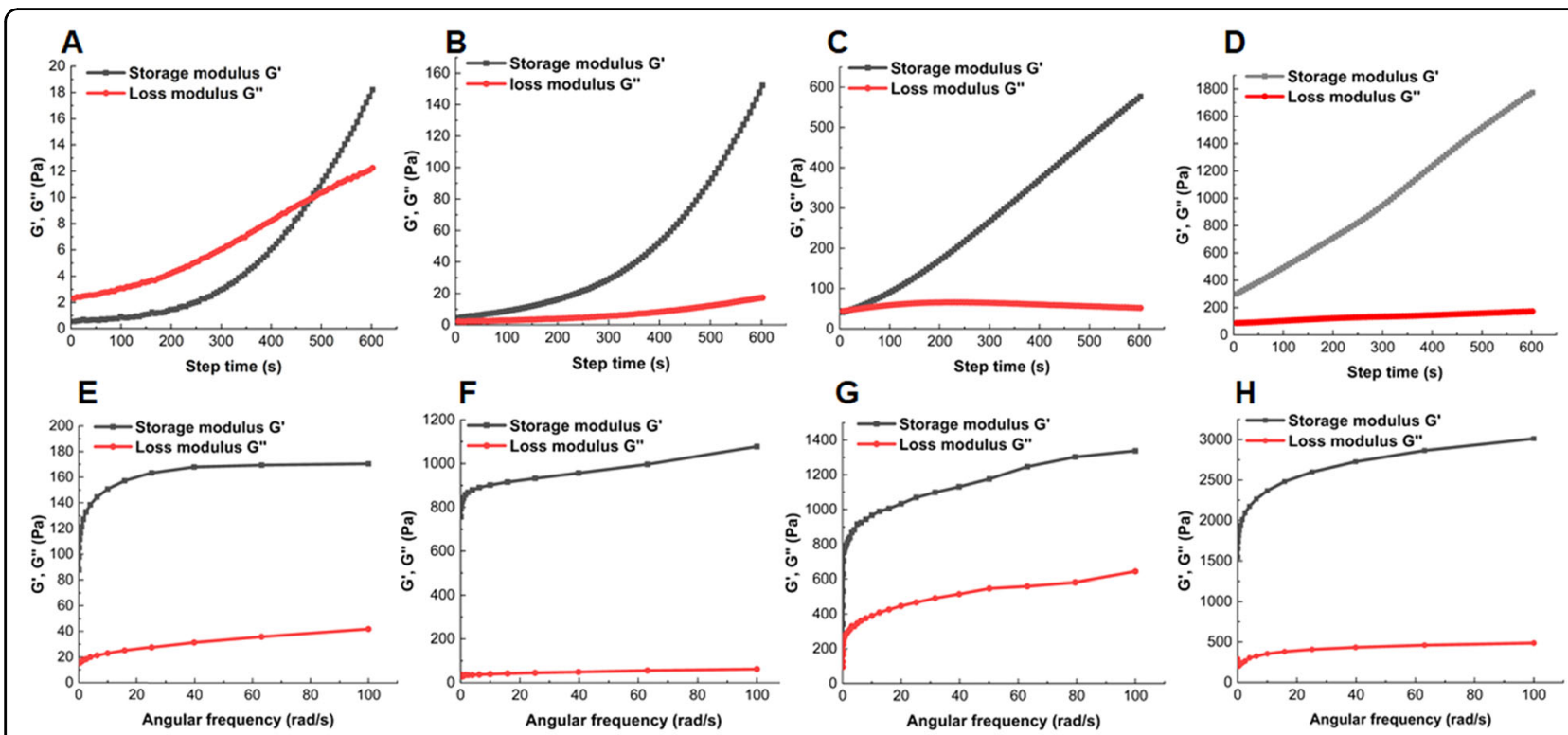

I
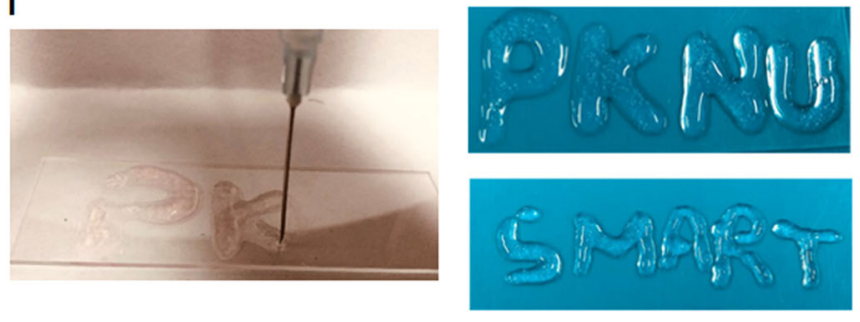

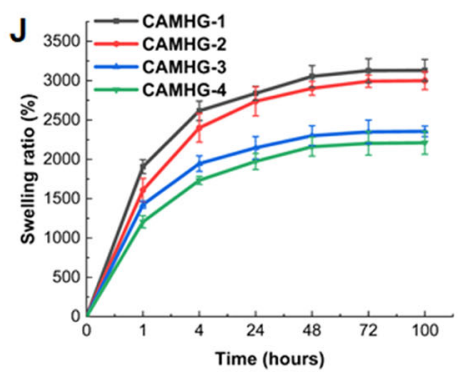

Fig. 4 Viscoelastic, injectability, and swelling characterization of hydrogels. A-D Storage modulus and loss modulus as a function of time for CAMHG-1, CAMHG-2, CAMHG-3, and CAMHG-4 hydrogels. E-H Storage modulus and loss modulus as a function of angular frequency for CAMHG-1, CAMHG-2, CAMHG-3, and CAMHG-4 hydrogels, I representative photographs showing injectability of hydrogels, and $\mathbf{J}$ swelling properties of hydrogels in PBS. 
prevail. This variation in the rates resulted in a $G^{\prime}$ and $G^{\prime \prime}$ "crossover", which is the time needed to reach gelation. On the other hand, hydrogels prepared from $4 \%$ CAM-Nb did not show the abovementioned crossover owing to their rapid gelation time (30-70 s). From Fig. 4A-D, it is also clear that a higher feed ratio of the cross-linker resulted in lower gelation times, suggesting that the crosslinker is the rate-limiting reagent during this initial state. In the frequency sweep graphs (Fig. 4E-H), G' was higher than $G^{\prime \prime}$ for CAM hydrogels with various concentrations, which showed that the CAM hydrogels have elastic behaviors. Specifically, the $G^{\prime}$ values of 2 and $4 \%$ CAM hydrogels at 10:10 feed ratios (CAMHG-2, CAMHG-4) were 1077 and $3012 \mathrm{~Pa}$, respectively, indicating a significant influence of the concentration of CAM-Nb on the mechanical properties of the hydrogels. Similarly, the mechanical strength of the hydrogels decreased when the molar ratio (10:5) of the cross-linker was decreased, resulting in $G^{\prime}$ values of 170 and $1338 \mathrm{~Pa}$ for hydrogels formulated from 2\% (CAMHG-1) and 4\% (CAMHG-3) $\mathrm{CAM}-\mathrm{Nb}$ solutions, respectively. These results revealed that the formulated hydrogels are injectable (as illustrated in Fig. 4I) and that the gelation times of the hydrogels can be simply adjusted by changing the percentage of the CAM-Nb precursor and the molar feed ratios of the cross-linker, as reported previously ${ }^{39}$.

\section{Swelling studies of hydrogels}

In this study, the \% swelling ratios (\%SR) of the hydrogels were analyzed by a gravimetric method. The \% SR of CAM-based hydrogels is shown in Fig. 4J. A dynamic increase in \%SR was observed during the first hour of immersion in PBS, followed by a steady increase to reach swelling equilibrium. Specifically, CAMHG-1, CAMHG-2, CAMHG-3, and CAMHG-4 hydrogels showed maximum swelling ratios of $3130 \%, 2999 \%$, $2356 \%$, and $2211 \%$ after $96 \mathrm{~h}$. It was revealed that swelling ratios were also influenced by the feed ratios of the crosslinker, as well as the concentration of CAM-Nb. The swelling ratio of the CAM hydrogels decreased by increasing the feed ratio of the cross-linker. The decrease in swelling ratio by increasing the molar amount of the cross-linker is a common phenomenon in hydrogels and might be attributed to the higher degrees of cross-linking, which subsequently decreased the mesh sizes and resulted in hydrogels with tighter structures ${ }^{40}$. This could also be caused by the limited free mobility of the macromolecular chains of the CAM, which did not allow water penetration. Therefore, hydrogels with higher degrees of crosslinking (CAMHG-2, CAMHG-4) swelled less than the same hydrogels with lower degrees of cross-linking (CAMHG-1, CAMHG-3). A similar trend was also observed when the concentration of $\mathrm{CAM}-\mathrm{Nb}$ was increased, i.e., the swelling ratio decreased when the concentration of CAM-Nb was increased (from $2 \%$ to $4 \%)$. This was also attributed to the highly dense or compact structures of the hydrogels at higher precursor concentrations. Overall, the swelling properties of CAM$\mathrm{Nb}$ hydrogels could be tuned by varying the concentration of the CAM-Nb precursor as well as that of the crosslinker.

\section{Drug loading and in vitro release studies}

To evaluate the reduction- and NIR light-responsive drug release behaviors of the CAM hydrogels, DOX, an anticancer agent belonging to the anthracycline antibiotics that suppresses or inhibits cancer cell growth via DNA cross-linking and free radical formation, was used as a model drug to be loaded into the CAM hydrogels during the formulation in PBS. The unloaded or surface-attached DOX was removed by washing with PBS after lyophilizing the hydrogels. The drug loading efficiency and drug loading contents were analyzed by using UV-visible spectrophotometry (Fig. S7). It was noted that CAM-Nb concentrations and the molar feed ratios of the crosslinker affected the drug loading efficiency. Specifically, CAMHG-1 and CAMHG-2 hydrogels (prepared from 2\% CAM-Nb, 10:5 and 10:10 feed ratios, respectively) showed drug loading efficiencies of $73 \pm 6 \%$ and $81 \pm 5 \%$, respectively (Table 1). On the other hand, CAMHG-3 and CAMHG-4 hydrogels revealed drug loading efficiencies of $89 \pm 2 \%$ and $93 \pm 3 \%$, respectively. The superior encapsulation efficiencies of the hydrogels obtained by increasing the CAM-Nb concentration and the feed ratio of the cross-linker could be ascribed to the firmly packed structures with interlocked cross-linked networks, which provide more efficient diffusion blockades to egress DOX from the hydrogels. These higher drug loading efficiencies could also be attributed to the presence of several negatively charged amino acids in the collagen and negatively charged GAGs of the CAM molecule, which might have tightly bound with the positively charged amine groups of the DOX molecules through electrostatic and/or ionic interactions.

The analysis of drug release from the CAM hydrogels was performed in a simulated physiological medium (PBS, $\mathrm{pH}$ 7.4) and in a simulated reducing medium $(10 \mathrm{mmol}$ GSH) mimicking the tumor microenvironment. DOX release was also carried out after exposure to NIR light. For the NIR light-responsive release behavior of DOX from the hydrogels, ICG, as a precursor of ROS, was also added into the hydrogels along with DOX. In PBS $(\mathrm{pH}$ 7.4), minimal DOX release without any apparent burst release was observed in all types of formulations of the CAM hydrogels, as shown in Fig. 5A. Specifically, after $96 \mathrm{~h}$, the CAMHG-1, CAMHG-2, CAMHG-3, and CAMHG-4 hydrogels showed 29.12\%, 25.01\%, 22.01\%, and $15.02 \%$ DOX release in PBS, respectively. On the 

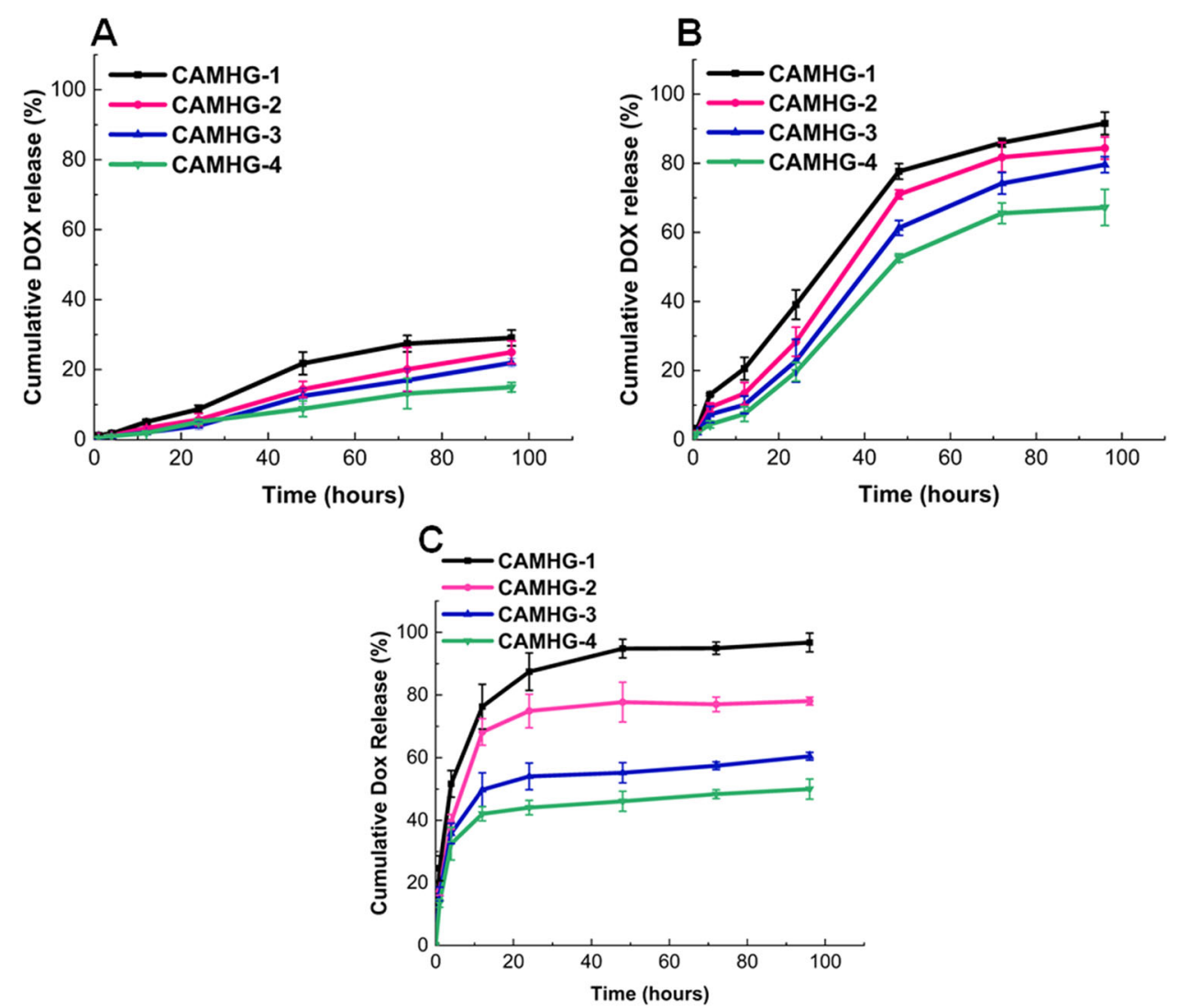

Fig. 5 Assessment of drug release from hydrogels under various conditions. A DOX release studies from hydrogels in simulated physiological buffer (PBS, pH =7.4), B DOX release studies in $10 \mathrm{mmol} G S H$, and C DOX release studies from DOX + ICG-loaded hydrogels in PBS after NIR exposure (2 W power, $15 \mathrm{~min})$. ICG (1 mg/mL) was added as a ROS precursor in NIR-responsive DOX release studies.

other hand, rapid release of DOX was noted when formulations were exposed to the reducing medium, as shown in Fig. 5B. Interestingly, DOX-loaded CAM hydrogels did not show any burst release in the reducing environment during the first $24 \mathrm{~h}$. This was followed by a steady and temporal drug release phase, where approximately $91.54 \%, 84.38 \%, 79.59 \%$, and $67.25 \%$ DOX release was observed after $96 \mathrm{~h}$ from the CAMHG-1, CAMHG-2, CAMHG-3, and CAMHG-4 hydrogels, respectively. In contrast, burst release of DOX was observed from the hydrogels during the first $4 \mathrm{~h}$ after exposure to NIR light, where $51.65 \%, 39.52 \%, 35.83 \%$, and $32.53 \%$ DOX release was noted from the CAMHG-1, CAMHG-2, CAMHG-3, and CAMHG-4 hydrogels, respectively (Fig. 5C). In the NIR-responsive release studies, the maximum DOX release was observed after $96 \mathrm{~h}$, where $96.72 \%$, 78.05\% $60.45 \%$, and $49.97 \%$ DOX release was noted from CAMHG-1, CAMHG-2, CAMHG-3, and CAMHG-4 hydrogels, respectively.

Notably, the initial 'burst release' from the CAM hydrogels upon exposure to NIR light was likely due to the rapid interaction of NIR light with the ICG molecules, generating ROS along with an increase in temperature, which robustly attacked and cleaved the $\mathrm{Se}-\mathrm{Se}$ bonds, resulting in the decross-linking or loosening of the gel structures and accelerating the release of the entrapped $\mathrm{DOX}^{41}$. The sustained DOX release phase was possibly due to DOX diffusion from the hydrogels into the release medium, as reported previously ${ }^{42}$. Typically, Se-Se bonds are oxidized to selenic acid in the presence of oxidants and reduced to selenol in a reducing medium ${ }^{43}$, furnishing diselenide moieties with dual-responsive drug release features.

\section{In vitro cytocompatibility of CAM, CAM-Nb, and hydrogels}

For biomedical usage of hydrogels, cytotoxicity assessment is essential, as biomaterials may cause unfavorable side effects in biological systems. In this study, the in vitro effects of CAM and the CAM-Nb precursor were assessed in HFF-1 fibroblast cells, while the cytocompatibility of the hydrogels was examined in human colorectal adenocarcinoma cells (HT-29 cells) using WST assays and further confirmed by live/dead assays. To evaluate cytocompatibility, fibroblast cells were incubated with various 

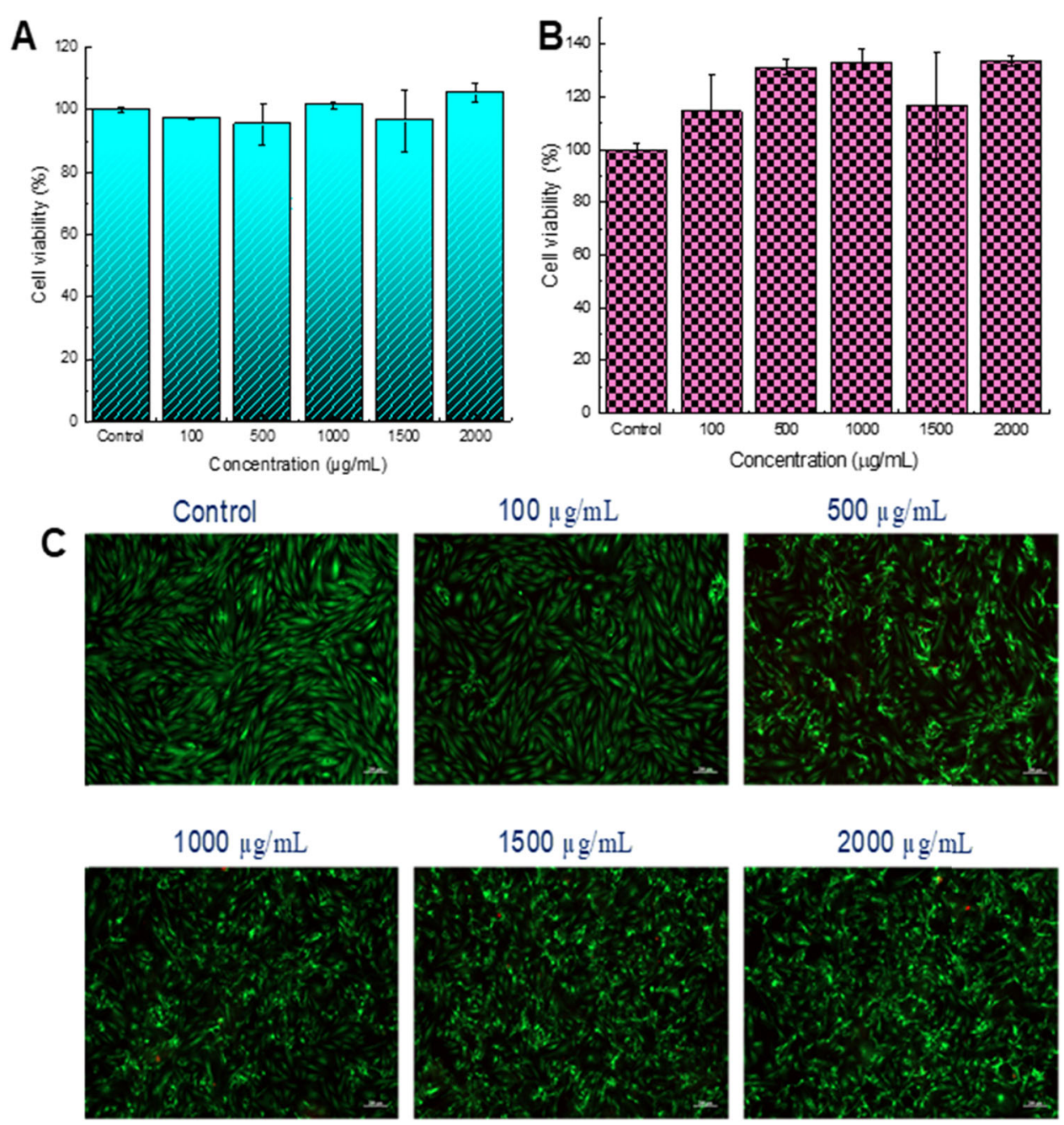

Fig. 6 Cytotoxicity studies of CAM and CAM-Nb precursors. A Cytotoxicity evaluation with various concentrations of CAM and B CAM-Nb. The cell line used was fibroblast cells (HFF-1). Cell viability was determined by WST cell proliferation assay. Error bars showing standard deviation $(n=4)$. C Confocal laser scanning micrographs showing the cytocompatibility of hydrogels in HFF-1 cells treated with various concentrations of CAM-Nb. Cell viability was determined by calcein-AM/ethidium homodimer-1 assay (live/dead assay). Green represents live cells, whereas red represents dead cells. Scale bars showing $200 \mu \mathrm{m}$.

doses of CAM and CAM-Nb for $48 \mathrm{~h}$. The WST assay data are shown in Fig. 6. Both CAM and CAM-Nb were cytocompatible up to a concentration of $2000 \mu \mathrm{g} / \mathrm{mL}$ ( $>90 \%$ cell viability), as shown in Fig. 6 A, B. Specifically, at the maximum tested concentration of $2000 \mu \mathrm{g} / \mathrm{mL}$, cells incubated with CAM and CAM-Nb showed cell viabilities of $105 \%$ and $133 \%$, respectively. The live/dead assays (Fig. $6 \mathrm{C})$ further confirmed the biocompatibility of CAM-Nb, where confocal micrographs showed highly viable cells (green color) without any dead cells (red color) after treatment with a maximum tested concentration of $2000 \mu \mathrm{g} / \mathrm{mL}$. These cytotoxicity studies indicated that CAM functionalization with the $\mathrm{Nb}$ moieties did not adversely affect the biocompatibility of the hydrogel precursor to be used for the development of biorthogonal injectable hydrogels.

Similarly, the in vitro cytocompatibility of the CAM hydrogels was evaluated by WST assays against HT-29 cells. The corresponding cell viabilities are shown in Fig. 7A. After treatment for $48 \mathrm{~h}$, the cells treated with the CAMHG-3 and CAMHG-4 hydrogels remained viable at over $100 \%$; however, significantly increased cell viabilities were shown after treatment with the CAMHG-1 and CAMHG-2 hydrogels. The increase in cell viability after treatment with CAMHG-1 and CAMHG-2 hydrogels could be due to the better porosity of these hydrogels, providing enhanced cell proliferation and microenvironments in the hydrogel scaffolds. Overall, these results 

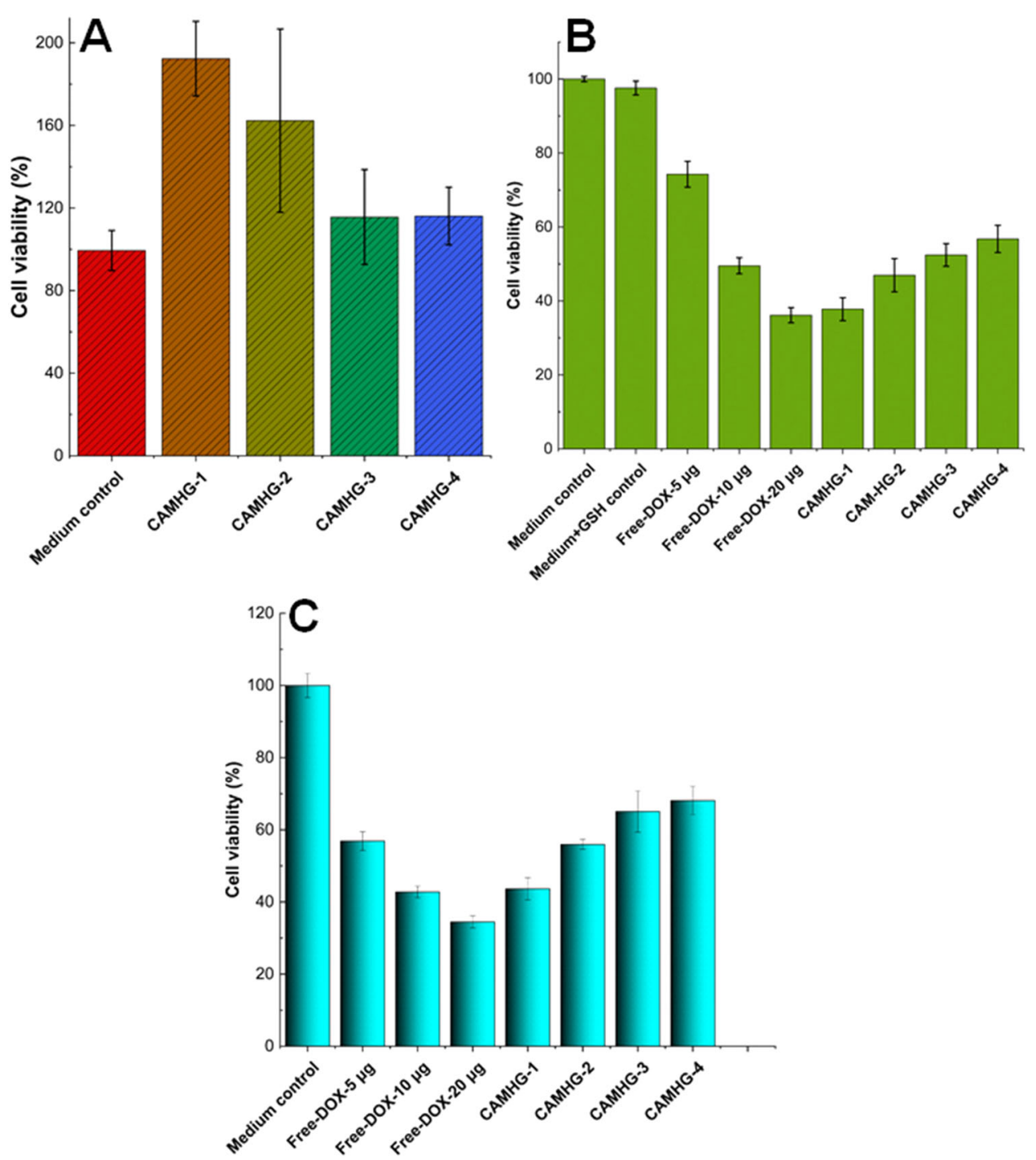

Fig. 7 Cytotoxicity assessment of hydrogels. A Cytocompatibility study of empty hydrogels in human colorectal adenocarcinoma cells (HT-29). Cell viabilities were determined by WST cell proliferation assays after incubation for $48 \mathrm{~h}$. B Cell viabilities of GSH treated HT-29 cells after treatment with free-DOX and DOX-loaded CAM hydrogels, and C cell viabilities of HT-29 cells after treatment with NIR irradiated (2-W power, $90 \mathrm{~s})$ free-DOX and DOX + ICG-loaded hydrogels. Error bars showing standard deviation $(n=4)$.

indicated that the prepared hydrogels did not show any apparent cytotoxicity; instead, cells proliferated well and exhibited better cytocompatibility compared to their control counterparts, indicating excellent biocompatibility and bioorthogonality of these CAM hydrogels. These results are consistent with previously reported ECMbased hydrogels, which were biocompatible with no apparent cytotoxicity and have been used in preclinical applications, for example, for the treatment of ulcerative colitis $^{44}$, traumatic brain injury ${ }^{45}$, and stroke ${ }^{46}$. The biocompatibility of the porcine omental-derived ECM hydrogel was also investigated in human-induced pluripotent stem cell-derived cardiomyocytes, and the results demonstrated good biocompatibility and no obvious cytotoxicity ${ }^{47}$.

\section{In vitro antitumor activity of DOX-loaded/DOX+ICG-loaded hydrogels}

The in vitro antitumor activity of DOX-loaded CAM hydrogels was assessed in HT-29 cells as a model cancer cell line by using WST assays. It was hypothesized that DOX-loaded CAM hydrogels will achieve antitumor effects similar to those of free DOX. For this purpose, GSH-treated HT-29 cells were cultured with all four types of hydrogels for $48 \mathrm{~h}$. Figure $7 \mathrm{~B}$ represents the in vitro antitumor activity of DOX-loaded CAM hydrogels and free 
DOX on GSH-treated HT-29 cells after treatment for $48 \mathrm{~h}$. The cell viabilities of the free DOX-treated cells were $60 \%$, $56 \%$, and $48 \%$ at DOX concentrations of 5,10 , and $20 \mu \mathrm{g}$, respectively. However, cell viabilities of $45 \%, 55 \%, 61 \%$, and $60 \%$ were noted with $20 \mu \mathrm{g}$ equivalents of DOX-loaded CAMHG-1, CAMHG-2, CAMHG-3, and CAMHG-4 hydrogels, respectively. From these data, it is apparent that the DOX-loaded CAMHG-1 hydrogel induced similarly in vitro antitumor activity ( $45 \%$ cell viability) compared to that of free DOX at the same concentration.

To evaluate NIR light-responsive DOX release and subsequent antitumor activity, DOX + ICG-loaded hydrogels were placed in the top chambers of the transwells, while HT-29 cells were cultured in the bottom chambers. Figure $7 \mathrm{C}$ shows the antitumor activities of DOX + ICG-loaded hydrogels after NIR exposure (2 W for $90 \mathrm{~s}$ ), which were slightly lower than that of free DOX. Specifically, cell viabilities of $62 \%, 64 \%, 74 \%$, and $78 \%$ were observed with $20 \mu \mathrm{g}$ equivalents of DOX-loaded CAMHG-1, CAMHG-2, CAMHG-3, and CAMHG-4 hydrogels, respectively. The difference in the antitumor activity of CAM hydrogels after treatment with GSH and NIR could be correlated with their different drug release patterns in GSH and NIR. Moreover, CAMHG-3 and CAMHG-4 hydrogels released comparatively lower amounts of DOX in response to both GSH and NIR stimuli after $48 \mathrm{~h}$ (Fig. 5B, C, respectively), therefore inducing lower antitumor activities. In contrast, CAMHG-1 and CAMHG-2 hydrogels released higher amounts of DOX after $48 \mathrm{~h}$, resulting in greater antitumor activity.

Moreover, redox potential is a frequently and extensively present phenomenon in physiological environments; normal cells retain a low level of redox potential, whereas redox progression in tumor cells could rise significantly ${ }^{19}$. Therefore, due to the reduction responsiveness and ICG-derived NIR light-triggered cleavage of the diselenide bonds, the prepared CAM-based injectable hydrogels could be used in dual-responsive drug delivery systems for tumor therapy. Generally, hyperthermia induced by photothermal agents upon specific NIR laser irradiation in the tumor region can not only kill cancer cells directly but also serve as a heat trigger to stimulate drug release in a controlled manner ${ }^{24,47}$. On the other hand, the optimized chemotherapy induced by external NIR light could help to completely eradicate tumors together with the internal GSH stimulus.

However, the utilization of CAM in drug delivery applications is still in the developmental stage. Specifically, a large portion of the current work still focuses on exploring the effects of different decellularization methods on the biochemical composition of CAM, the development of hydrogels, the characterization of their physical properties, and investigations in in vitro and in vivo animal models. At this time, clinical studies employing
CAM-derived highly porous hydrogels are very limited. While some examples of CAM-derived hydrogels have progressed to clinical trials, there is not a wealth of information on the clinical translation of these delivery strategies. However, by utilizing state-of-the-art techniques for the processing of CAM and the development of hydrogels, CAM-derived hydrogels have the potential to be used as a drug delivery system for clinical applications.

\section{Conclusions}

We developed injectable and highly porous hydrogels derived from CAM-Nb by using a novel diselenide-based water-soluble and reduction/NIR light dual-responsive cross-linker. The reaction between the $\mathrm{Nb}$ functionalities of the CAM and the Tz groups of the cross-linker evolved nitrogen gas and formed injectable hydrogels with highly porous structures. The hydrogel formulation showed adequate gelation times $(0.5-8 \mathrm{~min})$, offering the possibility of injecting them into the malignant site by a simple syringe injection, followed by solid gel formation. The porosities, swelling ratios, viscoelastic properties, drug loading, and release properties of the formulated injectable hydrogels could be tuned and controlled by simply adjusting the concentration of the CAM-Nb precursor and the feed ratios of the cross-linker. The formulated hydrogels released nominal amounts of DOX under a simplified (serum-free) physiological environment; however, sustained release of DOX was detected under reducing conditions, showing more than $90 \%$ DOX release after $96 \mathrm{~h}$. In contrast, NIR-irradiated ICG-loaded hydrogels exhibited burst release of DOX $(\sim 51 \%)$ during the first $4 \mathrm{~h}$, followed by a sustained release phase. Cytotoxicity assessments demonstrated that the CAM-Nb precursor and formulated hydrogels were cytocompatible with HFF-1 fibroblast cells and HT-29 human colon cancer cells, respectively. The DOX-loaded hydrogels inhibited the metabolic activity of HT-29 cells and induced antitumor effects that were comparable to those of free DOX. Overall, the data suggest that reduction and NIR dual-responsive CAM-based hydrogels could be promising candidates for tumorspecific internal stimuli (GSH)-responsive drug delivery, as well as for on-demand drug delivery after exposure to external stimuli (NIR light), to treat solid tumors.

\section{Acknowledgements}

This study was supported by National Research Foundation of Korea (NRF) Grants funded by the Ministry of Education (NRF-2018R1D1A3B07041437 and NRF-2019M3E5D1A02070861).

\section{Author details}

${ }^{1}$ Department of Smart Green Technology Engineering, Pukyong National University, Busan 48513, Republic of Korea. ${ }^{2}$ Department of Display Engineering, Pukyong National University, Busan 48513, Republic of Korea. ${ }^{3}$ Department of Biomedical Engineering, Pukyong National University, Busan 48513, Republic of Korea 


\section{Author contributions}

M.G. and S.-H.J. fabricated and functionalized the CAM. M.G. synthesized the crosslinker, formulated the hydrogels, and conducted the swelling, drug loading. and release analyses. S.-W.J and T.T.V. conducted the FTIR studies. S.-H.J. performed the rheology, cytocompatibility, and in vitro antitumor studies. K.T.L. and S.-H.P. designed and supervised the overall project. M.G. and K.T.L. cowrote the manuscript. All authors have read and approved the manuscript.

\section{Conflict of interest}

The authors declare no competing interests.

\section{Publisher's note}

Springer Nature remains neutral with regard to jurisdictional claims in published maps and institutional affiliations.

Supplementary information The online version contains supplementary material available at https://doi.org/10.1038/s41427-021-00354-4.

Received: 19 September 2021 Revised: 9 November 2021 Accepted: 7 December 2021.

Published online: 18 February 2022

\section{References}

1. El-Hussein, A., Manoto, S. L., Ombinda-Lemboumba, S., Alrowaili, Z. A. \& Mthunzi-Kufa, P. A review of chemotherapy and photodynamic therapy for lung cancer treatment. Anti-cancer Agents Med. Chem. 21, 149-161 (2021).

2. Chabner, B. A. \& Roberts, T. G. Chemotherapy and the war on cancer. Nat. Rev. Cancer 5, 65-72 (2005).

3. Nurgali, K, Jagoe, R. T. \& Abalo, R. Editorial: Adverse effects of cancer chemotherapy: anything new to improve tolerance and reduce sequelae? Front. Pharmacol. 9, 245-245 (2018).

4. Shih, H. \& Lin, C.-C. Photoclick hydrogels prepared from functionalized cyclodextrin and poly(ethylene glycol) for drug delivery and in situ cell encapsulation. Biomacromolecules 16, 1915-1923 (2015).

5. Buwalda, S. J. et al. Hydrogels in a historical perspective: from simple networks to smart materials. J. Control. Release 190, 254-273 (2014).

6. Yoshida, Y., Takahashi, A., Kuzuya, A. \& Ohya, Y. Instant preparation of a biodegradable injectable polymer formulation exhibiting a temperatureresponsive sol-gel transition. Polym. J. 46, 632-635 (2014).

7. Yan, S. et al. Injectable in situ self-cross-linking hydrogels based on poly(lglutamic acid) and alginate for cartilage tissue engineering. Biomacromolecules 15, 4495-4508 (2014).

8. Zhu, J. \& Marchant, R. E. Design properties of hydrogel tissue-engineering scaffolds. Expert Rev. Med. Devices 8, 607-626 (2011).

9. Frantz, C., Stewart, K. M. \& Weaver, V. M. The extracellular matrix at a glance. J. Cell Sci. 123, 4195-4200 (2010)

10. Fitzpatrick, L. E. \& McDevitt, T. C. Cell-derived matrices for tissue engineering and regenerative medicine applications. Biomater. Sci. 3, 12-24 (2015).

11. Nyambat, B. et al. New insight into natural extracellular matrix: Genipin crosslinked adipose-derived stem cell extracellular matrix gel for tissue engineering. Int. J. Mol. Sci. 21, 4864 (2020).

12. Fessel, G., Cadby, J., Wunderli, S., van Weeren, R. \& Snedeker, J. G. Dose- and time-dependent effects of genipin crosslinking on cell viability and tissue mechanics - toward clinical application for tendon repair. Acta Biomater. 10, 1897-1906 (2014).

13. Liang, Y. et al. Ph-responsive injectable hydrogels with mucosal adhesiveness based on chitosan-grafted-dihydrocaffeic acid and oxidized pullulan for localized drug delivery. J. Colloid Interface Sci. 536, 224-234 (2019).

14. Ohya, Y. Temperature-responsive biodegradable injectable polymer systems with conveniently controllable properties. Polym. J. 51, 997-1005 (2019).

15. Yang, F. et al. Injectable and redox-responsive hydrogel with adaptive degradation rate for bone regeneration. J. Mater. Chem. B 2, 295-304 (2014).

16. Wang, X., Wang, C., Zhang, Q. \& Cheng, Y. Near infrared light-responsive and injectable supramolecular hydrogels for on-demand drug delivery. Chem. Commun. 52, 978-981 (2016).
17. Ismail, Y. A., Martínez, J. G., Al Harrasi, A. S., Kim, S. J. \& Otero, T. F. Sensing characteristics of a conducting polymer/hydrogel hybrid microfiber artificial muscle. Sens. Actuators B Chem. 160, 1180-1190 (2011).

18. Zhao, Z. et al. Injectable postoperative enzyme-responsive hydrogels for reversing temozolomide resistance and reducing local recurrence after glioma operation. Biomater. Sci. 8, 5306-5316 (2020).

19. Gamcsik, M. P., Kasibhatla, M. S., Teeter, S. D. \& Colvin, O. M. Glutathione levels in human tumors. Biomarkers 17, 671-691 (2012).

20. Zhou, Y., Jie, K. \& Huang, F. A redox-responsive selenium-containing pillar [5]arene-based macrocyclic amphiphile: synthesis, controllable selfassembly in water, and application in controlled release. Chem. Commun. 53, 8364-8367 (2017).

21. Fleige, E., Quadir, M. A. \& Haag, R. Stimuli-responsive polymeric nanocarriers for the controlled transport of active compounds: concepts and applications. Adv. Drug Deliv. Rev. 64, 866-884 (2012).

22. Li, L., Scheiger, J. M. \& Levkin, P. A. Design and applications of photoresponsive hydrogels. Adv. Mater. 31, 1807333 (2019).

23. Chen, Y. et al. Antimony nanopolyhedrons with tunable localized surface plasmon resonances for highly effective photoacoustic-imaging-guided synergistic photothermal/immunotherapy. Adv. Mater. 33, 2100039 (2021).

24. Chen, S. et al. Eradication of tumor growth by delivering novel photothermal selenium-coated tellurium nanoheterojunctions. Sci. Adv. 6, eaay6825 (2020).

25. Qiu, M. et al. Biocompatible and biodegradable inorganic nanostructures for nanomedicine: silicon and black phosphorus. Nano Today 25, 135-155 (2019).

26. Luo, M., Fan, T., Zhou, Y., Zhang, H. \& Mei, L. 2d black phosphorus-based biomedical applications. Adv. Funct. Mater. 29, 1808306 (2019).

27. Wang, M. et al. NIR-triggered phototherapy and immunotherapy via an antigen-capturing nanoplatform for metastatic cancer treatment. Adv. Sci. $\mathbf{6}$, 1802157 (2019).

28. Kim, H. J. et al. In vivo degradation profile of porcine cartilage-derived extracellular matrix powder scaffolds using a non-invasive fluorescence imaging method. J. Biomater. Sci. Polym. Ed. 27, 177-190 (2016).

29. Park, D. Y. et al. Cross-linked cartilage acellular matrix film decreases postsurgical peritendinous adhesions. Artif. Organs 44, E136-E149 (2020).

30. Van Hoorick, J. et al. Cross-linkable gelatins with superior mechanical properties through carboxylic acid modification: Increasing the two-photon polymerization potential. Biomacromolecules 18, 3260-3272 (2017).

31. Guan, B., Yan, R., Li, R. \& Zhang, X. Selenium as a pleiotropic agent for medical discovery and drug delivery. Int. J. Nanomed. 13, 7473-7490 (2018).

32. Salma, S. A. et al. Near-infrared light-responsive, diselenide containing corecross-linked micelles prepared by the diels-alder click reaction for photocontrollable drug release application. Polym. Chem. 9, 4813-4823 (2018).

33. Oliveira, B. L., Guo, Z. \& Bernardes, G. J. L. Inverse electron demand diels-alder reactions in chemical biology. Chem. Soc. Rev. 46, 4895-4950 (2017).

34. Li, K., Fong, D., Meichsner, E. \& Adronov, A. Frontispiece: a survey of strainpromoted azide-alkyne cycloaddition in polymer chemistry. Chem. Eur. J. 27, 5057-5073 (2021)

35. Wang, J. et al. An injectable peg hydrogel controlling neurotrophin-3 release by affinity peptides. J. Control. Release 330, 575-586 (2021).

36. Siboro, S. A. P. et al. Tunable porosity of covalently crosslinked alginate-based hydrogels and its significance in drug release behavior. Carbohydr. Polym. 260, 117779 (2021).

37. Wang, L. et al. Fabrication of injectable, porous hyaluronic acid hydrogel based on an in-situ bubble-forming hydrogel entrapment process. Polymers 12, 1138 (2020).

38. Omidian, H., Rocca, J. G. \& Park, K. Advances in super porous hydrogels. J. Control. Release 102, 3-12 (2005).

39. Zhang, H., Huang, X., Jiang, J., Shang, S. \& Song, Z. Hydrogels with high mechanical strength cross-linked by a rosin-based crosslinking agent. RSC Adv. 7, 42541-42548 (2017).

40. Zhou, Z. et al. Effect of chemical cross-linking on properties of gelatin/hyaluronic acid composite hydrogels. Polym. Plast. Technol. Eng. 52, 45-50 (2013).

41. Anugrah, D. S. B., Ramesh, K., Kim, M., Hyun, K. \& Lim, K. T. Near-infrared light-responsive alginate hydrogels based on diselenide-containing crosslinkage for on demand degradation and drug release. Carbohydr. Polym. 223, 115070 (2019).

42. Li, J. \& Mooney, D. J. Designing hydrogels for controlled drug delivery. Nat. Rev. Mater. 1, 16071 (2016)

43. Ma, N., Li, Y., Xu, H., Wang, Z. \& Zhang, X. Dual redox responsive assemblies formed from diselenide block copolymers. J. Am. Chem. Soc. 132, 442-443 (2010). 
44. Keane, T. J. et al. Restoring mucosal barrier function and modifying macrophage phenotype with an extracellular matrix hydrogel: potential therapy for ulcerative colitis. J. Crohn's Colitis 11, 360-368 (2017).

45. $\mathrm{Wu}, \mathrm{Y}$. et al. Implantation of brain-derived extracellular matrix enhances neurological recovery after traumatic brain injury. Cell Transplant. 26, 1224-1234 (2017).
46. Ghuman, $\mathrm{H}$. et al. Long-term retention of ECM hydrogel after implantation into a sub-acute stroke cavity reduces lesion volume. Acta Biomater. 63, 50-63 (2017).

47. Chen, J. et al. Advances in nanomaterials for photodynamic therapy applications: status and challenges. Biomaterials 237, 119827 (2020). 